einer langen Zeit ganz allmählich in die Wohnkammern und in die übrigen Hoblräume des Kalksteins hineingepresst worden ist, und wir glauben, dass hierin auch ein Fingerzeig dafür gegeben ist, wie überhaupt in vielen Fällen die Petroleumlager entstanden sind. Der Sandstein und andere poröse Gesteine der Petroleumlager bilden die Hohlräume, in welche unter dem Druck der Erdschichten aus umgebenden bituminösen Schichten das Petroleum hineingedrückt worden ist, etwa wie aus einem öligen Niederschlag das Öl in eine darüber oder darunter befindliche poröse Thomplatte hineintritt oder hineingepresst werden kann.

\section{Ueber den Gebrauch einiger Indicatoren bei künstlicher Beleuchtung.}

(Mittheilung aus d. chem. Institut d. Landwirthsch. Akad. Bonn-Poppelsdorf.)

\section{Von Dr. A. Kufferath.}

Die im Folgenden dargelegten Versuche batten den 'Zweck, zu ermitteln, wie sich die zu Ammoniaktitrationen, z. B. zu Kjeldahlschen Stickstoffbestimmungen, geeigneten Indicatoren: Methylorange, Fluorescein, Cochenille, Corallin, p-Nitrophenol, Alizaringrïn B, Resazurin und Luteol bei känstlicher Beleuchtung in Bezug auf ihre Empfindlichkeit verhalten. Als Lichtquellen dienten eine elektrische Glühlampe von 16, eine Auerlampe von 13,5 und eine Fahrrad-Acetylenlampe von 12-12,5 Kerzenstärken.

Die Versuchsanordnung war so getroffen, dass einerseits dreimal je $10 \mathrm{~cm} 1 / 10$ N.Schwefelsäure abpipettirt und mit $50 \mathrm{ccm}$, $100 \mathrm{ccm}$ und $150 \mathrm{ccm}$ destillirtem ausgekochtem Wasser verdünnt, mittels 1/10 N.Kalilauge titrirt, andererseits drei Portionen von $1 / 10$ N.-Schwefelsäure mit je $100 \mathrm{ccm}$ destillirtem Wasser und steigenden Mengen von. chemisch reinem Chlorammonium, und zwar $0,5,1,5$ und $3,0 \mathrm{~g}$, vermischt, ebenfalls mit $1 / 10$ N.-Kalilauge titrirt wurden. Die Titrationen wurden in Erlenmeyer-Kolben von $450 \mathrm{ccm}$ Inhalt aus Jenaer Grerätheglas innerhalb der Temperaturgrenzen $15-17,5^{\circ} \mathrm{C}$. vorgenommen.

1. Methylorange. Aus einer heiss gesättigten, wässerigen Lösung wurde der Farbstoff des Handels mit Salzsäure gefällt, abfiltrirt und gut ausgewaschen, darauf in stark verdünntem Ammoniak gelöst und die ammoniakalische Lösung auf dem Wasserbade zur Trockne abgedampft; $1 \mathrm{~g}$ des so geroinigten Productes, in $500 \mathrm{ccm}$ aqua dest. gelöst, lieferte den Indicator. Ss hat den Anschein, als ob der gelblichere Ton des elektrischen Glühlichts im Vergleich zu Auer- und Acetylenlicht die Erkennung des Umschlags zu hellgelb etwas erschwere. Rein weisse Unterlage und Vergleich mit einem bei Tageslicht fertig titrirten Versuch erwiesen sich bei allen drei Lichtquellen als willkommene Hülfe. Für das Tageslicht fand ich die Zahlen:
$1 / 10 \mathrm{~N} \cdot \mathrm{SO}_{4} \mathrm{H}_{2}$
$\mathrm{H}_{2} \mathrm{O}$
$\mathrm{NH}_{4} \mathrm{Cl} \quad 1 / 10 \mathrm{~N}$.- $\mathrm{KOH}$
1. $10 \mathrm{ccm}+50 \mathrm{~cm}$
2. $10-+100$ -
3. $10-+150-$
4. $10-+100$
5. $10-+100$.
6. $10-+100$.

$$
=9,70 \text { - }
$$$$
=9,80 \mathrm{ccm}
$$$$
\begin{array}{r}
=9,70 \\
+0,59
\end{array}
$$$$
+1,5-=9,75
$$$$
+3,0-=9,75
$$

Mit diesen Zahlen stehen die bei Auerund Acetylenlichtbeleuchtung gewonnenen im Einklang; die gleiche Versuchsreihe bei Glühlicht ergab für:
1. $9,75-9,80$
2. $9,60-9,70$
3. $9,60-9,75$
4. $9,75-9,80$
5. $9,80-9,85$
6. $9,75-9,85$.

Für sämmtliche Titrationen reichten je zwei Tropfen Farbstofflösung von der oben angegebenen Concentration aus.

2. Fluoresceïn. Zur Bereitung der Indicatorlösung wurden $0,3 \mathrm{~g}$ reines Präparat in $100 \mathrm{ccm}$ Alkohol von 96 Proc. gelöst; zur Titration genügen hiervon $2-3$ Tropfen. Nach Vorschrift von Arnold ${ }^{x}$ wurde statt Kalilauge Ammoniak in Anwendung gebracht. Der Eintritt der Fluorescenz am Eude der Titration ist vorzüglich exkenubar auf tief schwarzer Unterlage; einer Vergleichsfarbe bedarf es nicht, wohl aber einer gewissen Vorübung des Auges. Bemerkenswerthe Unterschiede zwischen den einzelnen Lichtquellen und dem Tageslicht liessen sich nicht ermitteln. Bei letzterem fand ich:
$1 / 10 \mathrm{~N} .-\mathrm{SO}_{4} \mathrm{H}_{2} \quad \mathrm{H}_{2} \mathrm{O}$
1. $10 \mathrm{ccm}+50 \mathrm{ccm}$
2. $10-+100-$
$\mathrm{NH}_{4} \mathrm{Cl}$
$1 / 10 \mathrm{~N} \cdot \mathrm{NH}_{4} \mathrm{OH}$
3. $10-+150$ -
4. $10-+100=$
5. $10-+100$.
6. $10-+100$$$
=10,15-10,20 \mathrm{~cm}
$$$$
=10,05-10,15 \text { - }
$$$$
=10,05-10,15
$$$$
+0,5 \mathrm{~g}=10,05--10,20
$$$$
+1,5=10,10-10,20-
$$

Elektrisches Glühlicht gab für die gleichen 'Titrationen:
1. $10,10-10,20$
2. $10,10-10,20$
4. $10,10--10,15$
3. $10,15-10,20$
10,10-10,15
6. $10,15-10,20$.

Ganz analoge Ergebnisse lieferten die Versuche bei Auerlicht und Acetylenlicht.

3. Cochenille. Angewandt wurden zu jeder Titration $0,5 \mathrm{ccm}$ Cochenilletinctur des Handels. Die Titrationen wurden auf weisser Unterlage unter Heranziehung einer Vergleichsfarbe vorgenommen. Für Tageslicht fand ich:

1) Archiv de Pharmacie (3. R.) 94, 785. 


\begin{tabular}{|c|c|c|c|c|c|c|c|}
\hline 1. 10 & $\begin{array}{l}\mathrm{SO}_{4} \mathrm{H}_{2} \\
\mathrm{~cm}-1\end{array}$ & $\begin{array}{r}\mathrm{H}_{2} \\
+\quad 50\end{array}$ & $\mathrm{~cm}$ & $\mathrm{NH}_{4} \mathrm{Cl}$ & & $\begin{array}{l}1 / 10 \text {.N.- } \\
9,75\end{array}$ & $\begin{array}{l}\mathrm{BOH} \\
\mathrm{cmm}\end{array}$ \\
\hline 10 & $-\quad-1$ & +100 & - & & $=$ & 9,80 & - \\
\hline 10 & - & +150 & - & & $=$ & 9,80 & - \\
\hline 10 & - & +100 & - & $+0,5 \mathrm{~g}$ & $=$ & 9,80 & - \\
\hline 10 & - & +100 & - & $+1,5-$ & $=$ & 9,80 & - \\
\hline 6. 10 & - & +100 & - & $+3,0-$ & & 9,85 & - \\
\hline
\end{tabular}

Die gleichen Versuche bei elektrischem Glühlicht ergaben:
1. $9,70-9,85$
4. $9,80-9,90$
2. $9,70-9,85$
5. $9,80-9,95$
3. $9,75-9,90$
6. $9,70-9,85$;

d. h. der Umschlag von der hellen rosa Mischfarbe zum violett-rothen Farbenton braucht durchweg $0,10-0,15 \mathrm{ccm}^{1 / 10} \mathrm{~N}$.-KOH. Ebenso verhält sich der Indicator bei Auerlicht:
1. $9,75-9,85$
4. $9,75--9,85$
2. $9,80-9,95$
5. $9,80-9,90$
3. $9,85-9,95$
6. $9,80-9,90$.

Erheblich besser lässt sich aber der Umschlag erkennen bei Acetylenlicht:
1. $9,75-9,80$
4. $9,80-9,85$
2. $9,80-9,85$
5. $9,75-9,80$
3. $9,80-9,85$
6. $9,75-9,80$.

4. Corallin. Zu allen Titrationen wurden 6-7 Tropfen einer kalt gesättigten, wässerigen Farbstofflösung angewandt. Corallin erweist sich als einer der wenigst dankbaren Indicatoren zum Gebrauch bei künstlicher Beleuchtung. Mehr als böchstens $0,3 \mathrm{~g}$ Ammonsalze, eine Menge, die in praxi freilich nur selten in Frage kommt, mindern die Empfindlichkeit stark herab. Daher versetzte ich mit $0,1,0,2$ und $0,3 \mathrm{~g} \mathrm{NH}_{4} \mathrm{Cl}$ statt mit $0,5,1,5$ und $3,0 \mathrm{~g}$. Der Indicator scheint kohlensäureempfindlich zu sein; bläst man nämlich mit dem Munde durch eine mit $1 / 10$ N.-KOH fertig titrirte Menge von $10 \mathrm{ccm}$ $1 / 10 \mathrm{~N} .-\mathrm{SO}_{4} \mathrm{H}_{2}$ in $150 \mathrm{ccm}$ Wasser einige Secunden Luft durch eine.Glasröhre ein, so verschwindet der helle rosa Farbenton, und die Lösung wird wieder hellgelb. Bei Tageslicht fand ich die Zahlen:

\begin{tabular}{|c|c|c|c|c|c|c|}
\hline \multirow{2}{*}{\multicolumn{2}{|c|}{ 1. $\begin{array}{r}{ }^{1 / 10} \mathrm{~N} \cdot \mathrm{SO}_{4} \mathbf{H}_{2} \\
10 \mathrm{ccm}+\end{array}$}} & \multirow{2}{*}{\multicolumn{2}{|c|}{$\stackrel{\mathrm{H}_{2} \mathrm{O}}{ }$}} & \multicolumn{3}{|c|}{$1 / 10 \mathrm{~N}, \mathrm{KOH}$} \\
\hline & & & & & $=10,25$ & $\operatorname{ccm}$ \\
\hline 2. 10 & - & +100 & - & & $=10,20$ & - \\
\hline 3. 10 & - & +150 & - & & $=10,30$ & \\
\hline 4. 10 & - & +100 & - & $+0,1 \mathrm{~g}$ & $=10,05-10,25$ & - \\
\hline 5. 10 & - & +100 & - & $+0,2-$ & $=10,10-10,30$ & - \\
\hline 6. 10 & - & +100 & - & $+0,3-$ & $=10,00--10,40$ & - \\
\hline
\end{tabular}

Vergleichsflüssigkeit und weisse Unterlage erleichtern die Titration. Die gleiche Versuchsreihe ergab für elektrisches Glühlicht:
1. $10,20-10,25$
4. $10,20-10,45$
2. $10,20-10,30$
5. $10,30-10,50$
3. $10,30-10,40$
6. $10,30-10,55$

für Auerlicht:
1. $10,10--10,25$
2. $10,20-10,35$
4. $10,25-10,60$
3. $10,25-10,45$
$6.10,20-10,60$

Auch bei Corallin scheint mir das Acetylenlicht vor den übrigen zwei Beleuchtungsarten den Vorzug zu verdienen; die Grenzen, inner- halb deren der Umschlag klar erkennbar wird, rücken etwas näher aneinander. Bei denselben Versuchen wie oben fand ich für Acetylenlicht :
1. $10,20-10,25$
2. $10,30-10,35$
4. $10,20-10,35$
3. $10,30-10,40$
5. $10,20-10,35$
6. $10,20-10,45$.

5. p-Nitrophenol. Den zu titrirenden Säuremengen wurden je 6 Tropfen einer Lösung von $0,2 \mathrm{~g}$ chemisch reinem $\mathrm{p}$-Nitrophenol in $50 \mathrm{ccm}$ Alkohol von 96 Proc. zugesetzt. Durch seine Unempfindlichkeit gegen Ammonsalze sowohl, wie durch seine von L. Spiegel ${ }^{2}$ ) ermittelte Unempfindlichkeit gegen Kohlensäure, als Ersatz für Methylorange verwendbar, hat der Indicator noch den besonderen Vorzug, ohne Übergangsfarbe scharf von farblos zu hellgelb umzuschlagen. Dieser Umstand sichert auch bei ungeübtem Auge und bei allen drei Arten der künstlichen Beleuchtung gute Resultate. p-Nitrophenol scheint mir für Ammoniaktitrationen einer der besten Indicatoren zu sein. Der Umschlag erfolgte sowohl bei Tageslicht, als auch bei künstlicher Beleuchtung, auf rein weisser Unterlage leicht und sicher erkennbar, durch 1-2 Tropfen 1/, N.-Kalilauge. (4 Tropfen $=0,10 \mathrm{~cm}$ der zur Untersuchung gebrauchten Geissler'schen Bürette.)

6. Alizaringrün B von Dahl \& Co, Barmen. Verwendet wurde eine frisch bereitete, kalt gesättigte, wässerige Lösung des Farbstoffs. Die schon von J. Formánek ${ }^{3}$ ) beobachtete Empfindlichkeit des Indicators gegen Ammonsalze gestattete, ähnlich wie bei Corallin, nur einen Zusatz von reinem $\mathrm{NH}_{4} \mathrm{Cl}$ bis zu $0,4 \mathrm{~g}$. Der Umschlag ist auch für ein ungeübtes Auge durch das Fehlen störender Übergangstöne leicht zu erkennen. Die Titrationen bei Tageslicht ergaben:
$1 / 10 \mathrm{~N}^{-}-\mathrm{SO}_{4} \mathrm{H}_{2} \quad \mathrm{H}_{2} \mathrm{O}$
1. $10 \mathrm{~cm}+50 \mathrm{~cm}$
2. $10-+100-$
3. $10-+150$.
4. $10-+100-+0,15 \mathrm{~g}=9,90-10,00$ -
5. $10-+100-+0,25-=9,90-10,00$ -
6. $10-+100-+0,40-=9,95-10,00$

Dieselben Versuche gaben, bei Glühlicht wiederholt, die Zahlen:
1. 10,05
4. $10,00-10,25$
2. $10,00-10,05$
5. $10,00-10,30$
3. $9,95--10,05$
6. $9,90-10,30$.

Gegen die Versuche sub 1, 2 und 3 ist bei denjenigen mit Chlorammoniumzusatz der grüne Farbenton am Ende der Titration weniger feurig, mehr schmutzig-moosgrün und deshalb bei dem gelblicheren Glühlicht

2) Ber. 33, 2640.

3) Fres., Z. f. anal. Ch. 39, 99. 
etwas weniger gut erkennbar. Bei Auerlicht fand ich:
1. 9,95
4. $10,05-10,15$
2. 10,00
5. $10,10-10,30$
3. 10,05
6. $10,10-10,30$

bei Acetylenlicht:
1. 9,95
4. $10,10-10,20$
2. 10,10
5. $10,20-10,35$
3. 10,05
6. $10,20-10,35$

Das Acetylenlicht scheint mir auch hier, gleich wie bei Cochenille und Corallin, den Vorzug zu verdienen. Alizaringrün B ist anscheinend auch etwas kohlensäureempfindlich; Durchblasen von Luft mit dem Munde durch eine fertig titrirte Menge, Versuch 2 oder 3, mittels einer Glasröhre, lässt den grünen Farbenton wieder dem rothen weichen; kocht man die Flüssigkeit darauf eine halbe Minute, so tritt der umgekehrte Process ein, das Grün kommt wieder zum Vorschein.

7. Resazurin. Zur Darstellung des Indicators wurden $4 \mathrm{~g}$ Resorcin in $300 \mathrm{ccm}$ wasserfreiem Äther gelöst, mit einer geringen Menge conc. $\mathrm{NO}_{3} \mathrm{H}$, gesättigt mit salpetriger Säure, versetzt und einige Tage stehen gelassen, der abgeschiedene Farbstoff abfiltrirt und mit Äther gut ausgewaschen (Vorschrift von (rismer) ${ }^{4}$ ).

$0,2 \mathrm{~g}$ des Farbstoffs löste ich in $40 \mathrm{ccm}$ 1/10 N.- $\mathrm{NH}_{4} \mathrm{OH}$ und füllte mit destillirtem Wasser auf $1000 \mathrm{ccm}$ auf. Von dieser Lösung setzte ich den Tageslichttitrationen je $0,2 \mathrm{ccm}$, den übrigen $0,5 \mathrm{ccm}$ zu. Die Versuche bei Tageslicht lieferten die Resultate:

$$
\begin{aligned}
& 1 / 10 \mathrm{~N} \cdot \mathrm{SO}_{4} \mathrm{H}_{2} \quad \mathrm{H}_{2} \mathrm{O} \quad \mathrm{NH}_{4} \mathrm{Cl} \quad 1 / 10 \mathrm{~N} \cdot \mathrm{KOH} \\
& \text { 1. } 10 \mathrm{~cm}+50 \mathrm{ccm} \quad=9,75 \quad \mathrm{ccm} \\
& \text { 2. } 10-+100-\quad=9,80 \text { - } \\
& \begin{aligned}
& \text { 3. } 10-+150-=9,80 \\
& \text { 4. } 10-+100-+0,5 \mathrm{~g}=9,80-9,90
\end{aligned} \\
& \text { 5. } 10-+100-+1,5-=9,90-9,95- \\
& \text { 6. } 10-+100-+3,0-=9,85-9,95 \text {. }
\end{aligned}
$$

Auch für Glühlicht und Auerlicht ist der Umschlag noch genügend scharf: er erfolgt stets innerhalb 9,80-10,00; aber auch hier scheint das Acetylenlicht den beiden anderen Lichtarten überlegen; der Umschlag erfolgt innerhalb $0,05 \mathrm{ccm}^{1} / 10 \mathrm{~N}$.-Lauge. Ich fand für
1. $9,85-9,90$
4. $9,80-9,85$
2. $9,95-10,00$
5. $9,85-9,90$
3. $9,85-9,90$
6. $9,95-10,00$

die Versuche wurden auf weisser Unterlage ohne Vergleichsfarbe angestellt.

8. Luteol (Autenrieth). $1 \mathrm{~g}$ reines Präparat von Merck, Darmstadt, wurde in $300 \mathrm{ccm}$ absolutem Alkohol gelöst und in dicht schliessender Flasche aufgehoben. 2. Tropfen Farbstofflösung reichen für eine Titration aus. Der Indicator verhält sich wie $p$-Nitrophenol, dem er an Empfindlichkeit gleichsteht. Einen Unterschied in den

4) Fres., Z. f. anal. Ch. 37, 118.
Resultaten bei den drei Lichtquellen konnte ich nicht finden. Bei gleicher Versuchsanordnung wie sub 7 (Resazurin) fand ich bei Tageslicht für:
1. 9,85
4. 9,85
2. 9,90
5. 9,80
3. 9,85
6. 9,85 ;

bei Glühlicht für:
1. 9,95
4. 10,00
2.) 9,95
5. 10,05
6. 10,00 ;

bei Auerlicht für:
1. 2.$\} 9,95$
4. 9,90
5.) 9,95

bei Acetylenlicht für:
1. 9,90
4. 9,90
2. 9,95
5. $9,95-10,00$.

Luteol scheint etwas kohlensäureempfindlich zu sein.

Auf Grund der angeführten Versuche kam ich zu der Ansicht, dass das Acetylenlicht bei denjenigen Indicatoren Empfehlung verdient, welche zwischen zweierlei Farben umschlagen, also Methylorange, Cochenille, Corallin, Alizaringrün B und Resazurin, dass hingegen die Wahl der Lichtquelle von untergeordneter Bedeutung ist, wenn der Umschlag in anderer Weise erfolgt, wie bei p-Nitrophenol und Luteol von farblos zu hellgelb und bei Fluorescein von hellgelb zu gelbgrüner Fluorescenz.

\section{Fragen des gewerblichen Rechtsschutzes in der chemischen Industrie. Von Dr. Julius Ephraim. \\ [Schluss von S. 90:]}

3. Die Verfahren zur Herstellung von $\mathrm{Z}$ wischenproducten.

In der Industrie der Theerfarbstoffe nehmen die sogenannten Zwischenproducte, "die als Hülfsproducte bez. Durchgangsproducte für weitere chemische Verfahren Verwendung findenden " Stoffe eine ausserordentlich bedeutsame Rolle ein. Es ist allgemein bekannt, wie gerade die Industrie der Azofarbstoffe infolge der im Laufe der Zeit aufgefundenen Naphtolsulfosäuren, Dioxynaphtalinsulfosäuren, Naphtylaminsulfosäuren, Amidonaphtolsulfosäuren etc. vielfach neve Wege eingeschlagen hat. Die Wichtigkeit der Zwischenproducte tritt weiter in der Geschichte der künstlichen Darstellung des Indigos besonders deutlich hervor. Soweit es sich bei den Verfahren zur Darstellung von Zwischenproducten um Analogieverfahren handelt, also um Anwendungen von Methoden, 